\title{
Análise comparativa da ictiofauna da zona de arrebentação de praias arenosas do Estado de São Paulo, Brasil
}

\author{
Roberto GIANNINI ${ }^{1}$ \& Alfredo Martins PAIVA FILHO ${ }^{2}$ \\ ${ }^{1}$ Fundação de Estudos e Pesquisas Aquáticas (FUNDESPA) \\ ${ }^{2}$ Instituto Oceanográfico da Universidade de São Paulo \\ (Caixa Postal 66149, 05389-970 Sāo Paulo, SP, Brasil)
}

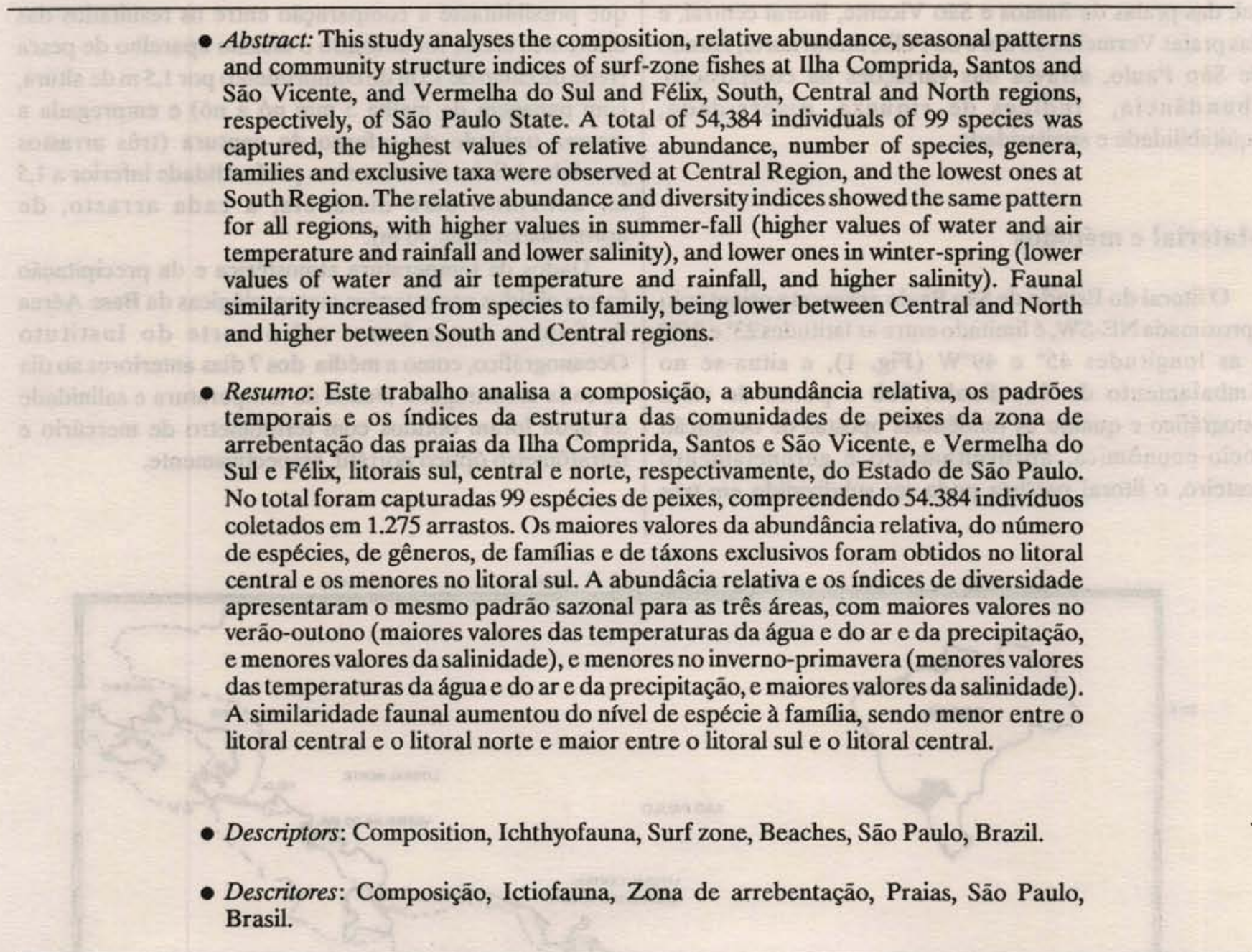

\section{Introdução}

A zona de arrebentação de Praias Arenosas, considerada como a faixa litorânea que se estende desde a linha de costa até o limite externo das células de circulação das correntes geradas pela própria ação das ondas (McLachlan \& Erasmus, 1983), é um ambiente altamente dinâmico, vulnerável e sujeito à extremas variaçōes ambientais. Apresenta uma variada fauna, principalmente

Contr. $n^{2} 790$ do Inst. oceanogr. da Usp. de zooplâncton e peixes, oferecendo uma série de vantagens aos organismos residentes, principalmente peixes e crustáceos juvenis, tais como maior proteção contra os predadores e maior abundância de alimento. A elevada diversidade e a abundância de sua fauna confirmam o relevante papel desempenhado pela zona de arrebentação como um criadouro natural e área de crescimento de várias espécies de peixes e crustáceos de interesse comercial e/ou esportivo (Anderson $\mathrm{Jr}$ et al., 1977; McLachlan \& Erasmus, 1983; Brown \& McLachlan, 1990). 
Devido à grande mobilidade, peixes sāo importantes como transformadores e exportadores de energia do sistema praia/zona de arrebentaçāo (Brown \& McLachlan, 1990). A comunidade ictiofaunística da zona de arrebentação de praias arenosas é muito dinâmica, com pequeno número de espécies dominantes e residentes e com variação sazonal na abundância (Anderson $\mathrm{Jr}$ et al., 1977; Saloman \& Naughton, 1979; Modde \& Ross, 1981; Cunha, 1981; Monteiro Neto, 1990).

O presente trabalho, síntese do estudo de Giannini (1994), objetivou analisar a fauna de peixes da zona de arrebentação da praia de Fora da Ilha Comprida, litoral sul, das praias de Santos e São Vicente, litoral central, e das praias Vermelha doSul e do Félix, litoral norte, Estado de São Paulo, através das variações na composição, abundância, índices de riqueza, diversidade, eqüitabilidade e similaridade.

\section{Material e métodos}

O litoral do Estado de Sāo Paulo apresenta orientação aproximada NE-SW, é limitado entre as latitudes $23^{\circ}$ e $25^{\circ} \mathrm{S}$ e as longitudes $45^{\circ}$ e $49^{\circ} \mathrm{W}$ (Fig. 1), e situa-se no Embaiamento de São Paulo. Sob o ponto de vista fisiográfico e quanto às tendências opostas de ocupaçāo sócio-econômica, aproveitamento e gerenciamento costeiro, o litoral paulista pode ser subdividido em três áreas que apresentam características diversas: litoral sul, do extremo sul do Estado até Praia Grande, litoral central, caracterizado pela Baixada Santista, e litoral norte, de Bertioga até o extremo norte do Estado.

As amostras foram provenientes de capturas quinzenais na praia de Fora da Ilha Comprida, entre julho de 1990 e julho de $1991 \mathrm{em} 10$ estaçōes fixas, mensais nas praias de Santos e São Vicente, de março de 1985 a maio de 1986 em 13 estaçōes fixas, e mensais nas praias Vermelha do Sul e do Félix, entre julho de 1988 e junho de 1989 em 1 estação fixa em cada praia (Fig. 1).

A fim de obter-se uma padronização nas amostragens, que possibilitasse a comparação entre os resultados das diferentes áreas, foi utilizado o mesmo aparelho de pesca (rede de calão de $15 \mathrm{~m}$ de comprimento por $1,5 \mathrm{~m}$ de altura, com panagem de malha $5 \mathrm{~mm}$ nó a no) e empregada a mesma unidade de esforço de captura (três arrastos paralelos à linha de costa e em profundidade inferior a 1,5 m, cobrindo uma distância, a cada arrasto, de aproximadamente $50 \mathrm{~m}$ ).

Dados da temperatura atmosférica e da precipitação foram obtidos nas estaçōes meteorológicas da Base Aérea de Santos e nas bases sul e norte do Instituto Oceanográfico, como a média dos 7 dias anteriores ao dia de cada amostragem. Dados da temperatura e salinidade da água foram obtidos com termômetro de mercúrio e refratômetro 6 ptico portátil, respectivamente.

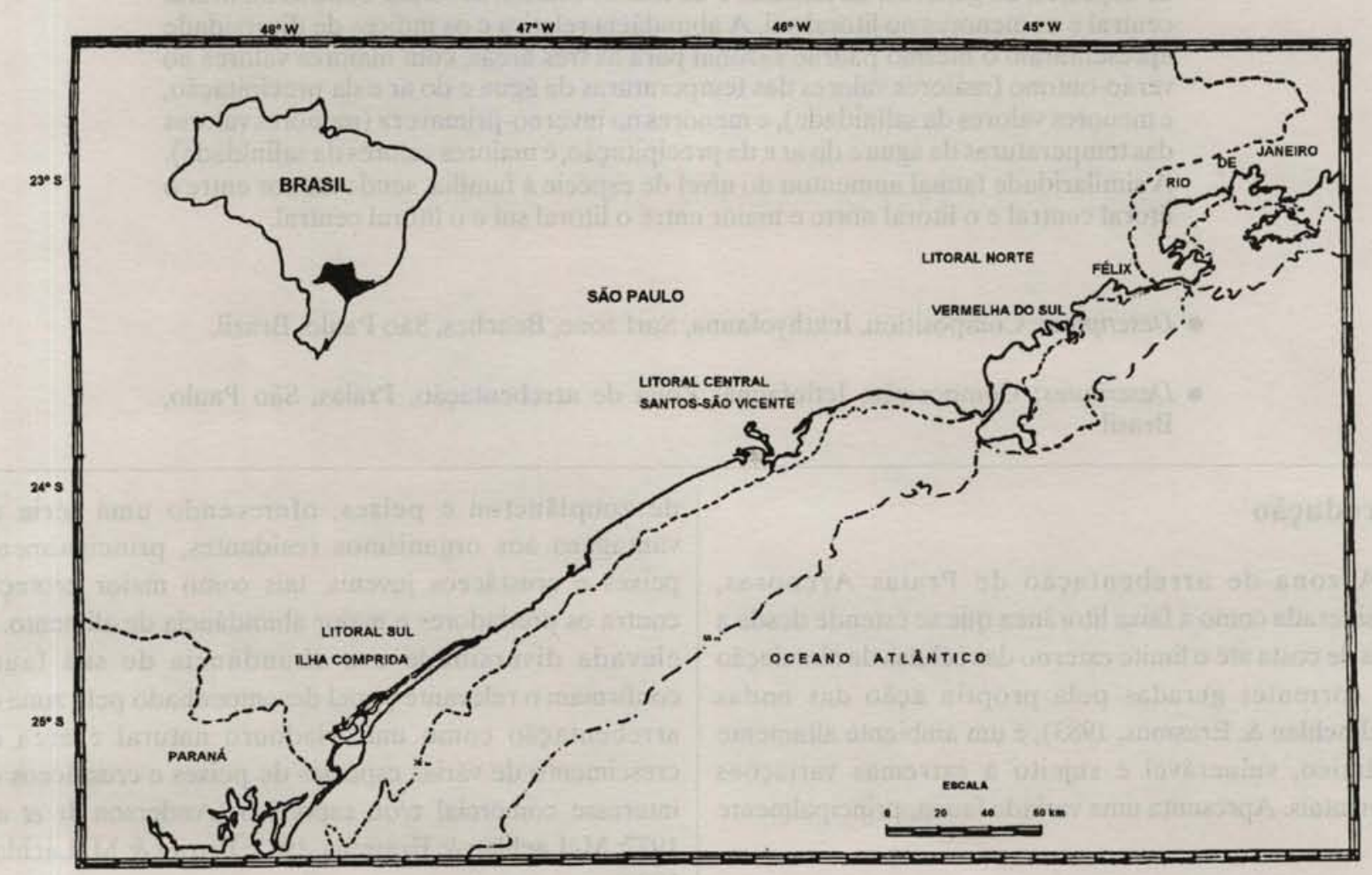

Fig. 1. Mapa do litoral do Estado de São Paulo, com a indicação das áreas de amostragem. 
Todos os exemplares capturados foram fixados em formalina a $10 \%$ ou congelados no local. Em laboratório, todos os peixes foram identificados, com o auxílio dos trabalhos de Norman (1934), Figueiredo (1977), Figueiredo \& Menezes (1978, 1980), Fischer (1978) e Menezes \& Figueiredo $(1980,1985)$, contados, pesados e medidos no comprimento total.

Foram determinados o número de famílias, gêneros, espécies (S) e exemplares ocorrentes, a composiçāo percentual em número das familias mais abundantes, a variação da captura total por unidade de esforço (CPUE), e a variação total do comprimento médio dos exemplares. Para cada espécie, foi determinada a abundância numérica e a freqüência de ocorrência, em termos absolutos e percentuais.

Foram determinados também os índices de Riqueza de Espécies (D), de Diversidade de Shannon (H'), e de Eqüitabilidade (J'), calculados por amostra, como o valor médio.

Os valores dos parâmetros acima mencionados foram expressos como valores médios por área de amostragem e por estação do ano.

A comparação ictiofaunística entre as áreas de amostragem foi realizada pela análise básica dos fatores abióticos e da abundância relativa dos indivíduos capturados, e pela determinação das similaridades e variabilidades na composiçẩo das comunidades ao nível de espécie, gênero e familia, através do índice de similaridade de Jaccard.

O número total de espécies, gêneros e famílias que ocorreram em cada uma das áreas de amostragem do presente estudo foi tabulado, sendo também expressa a percentagem dos táxons de acordo com o critério de ocorrência em cada área. Foi determinado, também, o número de espécies, gêneros e famílias exclusivas e comuns entre as áreas de amostragem e a percentagem de cada um destes à abundância total.

\section{Resultados}

Analisando-se as três áreas, verificamos que os parâmetros ambientais registrados apresentaram o mesmo padrâo sazonal, com menores valores das temperaturas da água e do ar, da precipitação e os maiores valores da salinidade no inverno-primavera e os maiores valores das temperaturas da água e do ar, da precipitaçāo e os menores valores da salinidade no verão-outono. Os maiores valores e a pequena variação sazonal da salinidade observada no litoral norte refletem a ausência de aporte d'água continental significativo nesta área de estudo. À exceção da primavera-verâo no litoral norte, os valores da temperatura da água foram superiores aos da temperatura do ar, principalmente durante os meses de verão-outono, diminuindo essa diferença nos meses de inverno (Fig. 2).

No total foram capturados 54.384 indivíduos, em 1.275 arrastos (média ligeiramente inferior a $\mathbf{4 3}$ indivíduos por arrasto) efetuados em 425 amostras. A captura média por unidade de esforço foi maior no litoral central, superando em mais de quatro vezes a captura média do litoral sul e em mais de duas vezes a captura média do litoral norte, sendo inclusive superior à média global (Tab. 1).
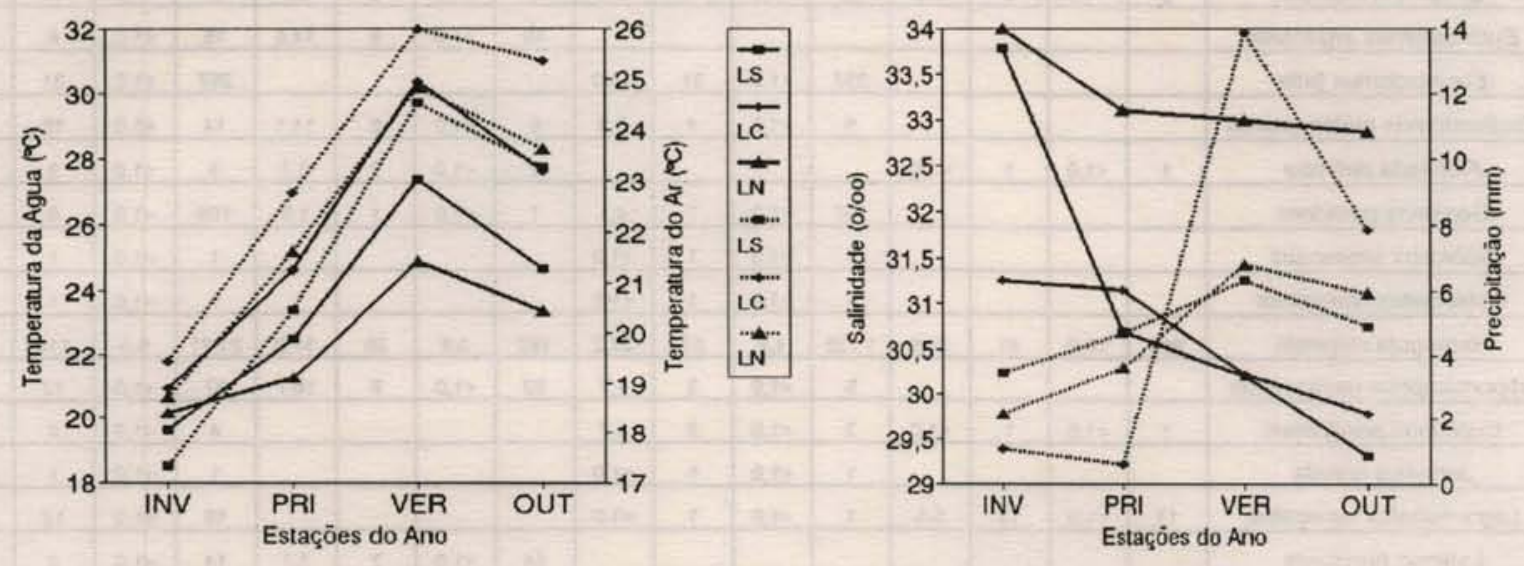

Fig. 2. Variação sazonal da temperatura e da salinidade da água (linha cheia), e da temperatura atmosférica eda precipitação (linha pontilhada), no litoral sul (LS), central (LC) e norte (LN). 
Tabela 1. Abundância numérica absoluta $(N)$ e percentual $(\% N)$, frequeência de ocorrência absoluta $(F)$ e percentual (\%F), das espécies de peixes capturadas no litoral sul (LS), central (LC), norte (LN) e no Estado de São Paulo (SP)

\begin{tabular}{|c|c|c|c|c|c|c|c|c|c|c|c|c|c|c|c|c|}
\hline & \multicolumn{4}{|c|}{ LS } & \multicolumn{4}{|c|}{ LC } & \multicolumn{4}{|c|}{ LN } & \multicolumn{4}{|c|}{ SP } \\
\hline ESPÉCIE & $\mathrm{N}$ & $\% \mathrm{~N}$ & $F$ & $\% \mathrm{~F}$ & $\mathrm{~N}$ & $\% \mathrm{~N}$ & $\mathrm{~F}$ & $\% \mathrm{~F}$ & $\mathrm{~N}$ & $\% \mathrm{~N}$ & $\mathrm{~F}$ & $\% \mathrm{~F}$ & $\mathrm{~N}$ & $\% \mathrm{~N}$ & $\mathrm{~F}$ & $\% \mathrm{~F}$ \\
\hline Anchoa filifera & & & & & 1.308 & 3,3 & 14 & 8,2 & & & & & 1.308 & 2,4 & 14 & 3,3 \\
\hline Anchoa januaria & 11 & $<1,0$ & 10 & 5,0 & & & & & 3 & $<1,0$ & 1 & 1,9 & 14 & $<1,0$ & 11 & 2,6 \\
\hline Anchoa lyolepis & 2 & $<1,0$ & 1 & $<1,0$ & & & & & 12 & $<1,0$ & 3 & 5,6 & 14 & $<1,0$ & 4 & $<1,0$ \\
\hline Anchoa marinii & 27 & $<1,0$ & 11 & 5.5 & 10 & $<1,0$ & 2 & 1,2 & 123 & 2,3 & 10 & 18.5 & 160 & $<1,0$ & 23 & 5,4 \\
\hline Anchoa tricolor & 2 & $<1,0$ & 1 & $<1,0$ & 1 & $<1,0$ & 1 & $<1,0$ & 484 & 9,1 & 8 & 14,8 & 487 & $<1,0$ & 10 & 2.3 \\
\hline Anchoviella brevirostris & 18 & $<1,0$ & 2 & 1,0 & & & & & & & & & 18 & $<1,0$ & 2 & $<1,0$ \\
\hline Anchoviella lepidentostole & 116 & 1.2 & 21 & 10,6 & 10.691 & 27,1 & 106 & 61,6 & & & te & & 10.807 & 19,9 & 127 & 29,9 \\
\hline Anisotremus surinamensis & & & & & & & & & 1 & $<1,0$ & 1 & 1,9 & 1 & $<1,0$ & 1 & $<1,0$ \\
\hline Archosargus momboidalis & & & & & & & & & 1 & $<1,0$ & 1 & 1,9 & 1 & $<1,0$ & 1 & $<1,0$ \\
\hline Astroscopus ygraecum & 3 & $<1,0$ & 2 & 1,0 & & & & & 1 & $<1,0$ & 1 & 1,9 & 4 & $<1,0$ & 3 & $<1,0$ \\
\hline Bagre bagre & 1 & $<1.0$ & 1 & $<1.0$ & & & & & & & & & 1 & $<1.0$ & 1 & $<1,0$ \\
\hline Bathygobius soporator & & & & & 8 & $<1,0$ & 5 & 2,9 & & & & & 8 & $<1,0$ & 5 & 1,2 \\
\hline Caranx hippos & 16 & $<1,0$ & 5 & 2,9 & 8 & $<1,0$ & 5 & 2.5 & & & & & 24 & $<1,0$ & 10 & 2,3 \\
\hline Caranx latus & 31 & $<1,0$ & 15 & 7,5 & 47 & $<1,0$ & 18 & 10,5 & 9 & $<1,0$ & 7 & 13.0 & 87 & $<1,0$ & 40 & 9.4 \\
\hline Cathorops spixii & & & & & 1 & $<1,0$ & 1 & $<1,0$ & & & & & 1 & $<1,0$ & 1 & $<1,0$ \\
\hline Centropomus parallelus & 1 & $<1,0$ & 1 & $<1,0$ & & & & & & & & & 1 & $<1,0$ & 1 & $<1,0$ \\
\hline Centropomus undecimalis & 6 & $<1,0$ & 5 & 2,5 & & & & & & & & & 6 & $<1,0$ & 5 & 1,2 \\
\hline Cetengraulis edentulus & 76 & $<1,0$ & 8 & 4,0 & 2 & $<1,0$ & 2 & 1,2 & & & & & 78 & $<1,0$ & 10 & 2,3 \\
\hline Chaetodiptenis faber & 4 & $<1,0$ & 3 & 1,5 & 6 & $<1,0$ & 5 & 2,9 & & & & & 10 & $<1,0$ & 8 & 1,9 \\
\hline Chilomycterus spinosus & 5 & $<1,0$ & 5 & 2,5 & 1 & $<1,0$ & 1 & $<1,0$ & & & & & 6 & $<1,0$ & 6 & 1,4 \\
\hline Chirocentrodon bleekerianus & & & & & & & & & 8 & $<1,0$ & 1 & 1,9 & 8 & $<1,0$ & 1 & $<1,0$ \\
\hline Chloroscombrus chrysurus & 127 & 13 & 12 & 6,0 & 28 & $<1,0$ & 6 & 3,5 & 28 & $<1,0$ & 7 & 13,0 & 183 & $<1,0$ & 25 & 5,9 \\
\hline Citharichthys arenaceus & 91 & $<1,0$ & 25 & 12,6 & & & & & 1 & $<1,0$ & 1 & 1,9 & 92 & $<1,0$ & 26 & 6,1 \\
\hline Citharichthys spilopterus & 4 & $<1,0$ & 4 & 2,0 & & & & & & & & & 4 & $<1,0$ & 4 & $<1,0$ \\
\hline Conodon nobilis & 3 & $<1,0$ & 2 & 1,0 & 22 & $<1,0$ & 11 & 6,4 & 7 & $<1,0$ & 4 & 7,4 & 32 & $<1,0$ & 17 & 4,0 \\
\hline Cynoscion leiarchus & 2 & $<1,0$ & 2 & 1,0 & & & & & & & & & 2 & $<1,0$ & 2 & $<1,0$ \\
\hline Dactyloscopus tridigitatus & & & & & & & & & 71 & 1,3 & 23 & 42,6 & 71 & $<1,0$ & 23 & 5,4 \\
\hline Diaptenus mombeus & 1 & $<1,0$ & 1 & $<1,0$ & & & & & 1 & $<1,0$ & 1 & 1,8 & 2 & $<1,0$ & 2 & $<1,0$ \\
\hline Diplectrum radiale & & & & & 2 & $<1,0$ & 1 & $<1,0$ & & & & & 2 & $<1,0$ & 1 & $<1,0$ \\
\hline Diplodus argenteus & & & & & & & & & 120 & 2.3 & 4 & 7.4 & 120 & $<1,0$ & 4 & $<1,0$ \\
\hline Etropus crossotus & 2 & $<1,0$ & 2 & 1,0 & 43 & $<1,0$ & 15 & 8.7 & 2 & $<1,0$ & 2 & 3,7 & 47 & $<1,0$ & 19 & 4,5 \\
\hline Eucinostomus argenteus & & & & + & & & & & 16 & $<1,0$ & 8 & 14,8 & 16 & $<1,0$ & 8 & 1,9 \\
\hline Eucinostomus gula & & & 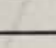 & $E$ & 257 & $<1,0$ & 31 & 18.0 & & & & & 257 & $<1,0$ & 31 & 7,3 \\
\hline Eucinostomus melanopterus & & & & 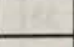 & 5 & $<1,0$ & 4 & 2,3 & 9 & $<1,0$ & 6 & 11,1 & 14 & $<1.0$ & 10 & 2,3 \\
\hline Fistularia petimba & 1 & $<1,0$ & 1 & $<1,0$ & & - & & & 2 & $<1,0$ & 2 & 3,7 & 3 & $<1,0$ & 3 & $<1,0$ \\
\hline Genidens genidens & & & & & 107 & $<1,0$ & 7 & 4,1 & 1 & $<1,0$ & 1 & 1,9 & 108 & $<1,0$ & 8 & 1,9 \\
\hline Gobiesox strumosus & & & & & 1 & $<1,0$ & 1 & $<1,0$ & & & 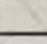 & + & 1 & $<1,0$ & 1 & $<1,0$ \\
\hline Gobionellus oceanicus & & & & & 1 & $<1,0$ & 1 & $<1,0$ & & & 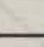 & & 1 & $<1,0$ & 1 & $<1,0$ \\
\hline Harengula clupeola & 964 & 10,0 & 41 & 20,6 & 1.725 & 4,4 & 57 & 33,2 & 192 & 3,6 & 20 & 37,0 & 2.881 & 5,3 & 118 & 27,8 \\
\hline Hyporhamphus unifasciatus & & & 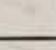 & & 5 & $<1,0$ & 3 & 1,7 & 32 & $<1,0$ & 9 & 16,7 & 37 & $<1,0$ & 12 & 2,8 \\
\hline Isopisthus parvipinnis & 1 & $<1,0$ & 1 & $<1,0$ & 3 & $<1,0$ & 3 & 1,7 & & & & & 4 & $<1,0$ & 4 & $<1,0$ \\
\hline Jenynsia lineata & & & & & 1 & $<1,0$ & 1 & $<1,0$ & & & & & 1 & $<1,0$ & 1 & $<1,0$ \\
\hline Lagocephalus laevigatus & 17 & $<1,0$ & 11 & 5,5 & 1 & $<1,0$ & 1 & $<1,0$ & & & & & 18 & $<1,0$ & 12 & 2,8 \\
\hline Larimus breviceps & & & & & & & & & 14 & $<1,0$ & 2 & 3.7 & 14 & $<1,0$ & 2 & $<1,0$ \\
\hline Lobotes surinamensis & 3 & $<1,0$ & 3 & 1.5 & & & & & & & & & 3 & $<1,0$ & 3 & $<1,0$ \\
\hline Menticirmus americanus & 49 & $<1,0$ & 12 & 6.0 & 282 & $<1,0$ & 15 & 8.7 & & & & & 331 & $<1,0$ & 27 & 6,4 \\
\hline Menticimhus littoralis & 2143 & 22,2 & 148 & 74.4 & 2870 & 7,3 & 155 & 90,1 & 197 & 3,7 & 27 & 50,0 & 5.210 & 9,6 & 330 & 77,6 \\
\hline Micropogonias furnieri & 40 & $<1,0$ & 8 & 4,0 & 708 & 1,8 & 38 & 22.1 & & & - & & 748 & 1,4 & 46 & 10,8 \\
\hline Mugil curema & 790 & 8,2 & 122 & 61,3 & 3.583 & 9,1 & 44 & 25,6 & & & & & 4.373 & 8.0 & 166 & 39,1 \\
\hline Mugil gaimardianus & 434 & 4.5 & 23 & 11,6 & 2.765 & 7,0 & 92 & 53,5 & 14 & $<1,0$ & 11 & 20,4 & 3.213 & 5.9 & 126 & 29,6 \\
\hline Mugil platanus & 5 & $<1,0$ & 3 & 1,5 & & & & & & & & & 5 & $<1,0$ & 3 & $<1,0$ \\
\hline Narcine brasiliensis & & & & & & & & & 1 & $<1,0$ & 1 & 1,9 & 1 & $<1,0$ & 1 & $<1,0$ \\
\hline
\end{tabular}


Tabela 1. Cont.

\begin{tabular}{|c|c|c|c|c|c|c|c|c|c|c|c|c|c|c|c|c|}
\hline & \multicolumn{4}{|c|}{ LS } & \multicolumn{4}{|c|}{ LC } & \multicolumn{4}{|c|}{ LN } & \multicolumn{4}{|c|}{ SP } \\
\hline ESPÉCIE & $\mathbf{N}$ & $\% \mathrm{~N}$ & $\mathrm{~F}$ & $\% \mathrm{~F}$ & $\mathrm{~N}$ & $\% \mathrm{~N}$ & $\mathbf{F}$ & $\% \mathrm{~F}$ & $\mathrm{~N}$ & $\% \mathrm{~N}$ & $\mathrm{~F}$ & $\% \mathrm{~F}$ & $\mathbf{N}$ & $\% \mathrm{~N}$ & $\mathbf{F}$ & $\% \mathrm{~F}$ \\
\hline Netuma barba & 115 & 1.2 & 17 & 8.5 & 1.302 & 3.3 & 42 & 24,4 & & & & & 1.417 & 2.6 & 59 & 13,9 \\
\hline Odontesthes bonariensis & 181 & 1,9 & 62 & 31,2 & 109 & $<1,0$ & 31 & 18,0 & 30 & $<1,0$ & 4 & 7.4 & 320 & $<1,0$ & 123 & 28,9 \\
\hline Oligoplites palometa & 3 & $<1,0$ & 2 & 1,0 & & & & & & & & & 3 & $<1,0$ & 2 & $<1,0$ \\
\hline Oligoplites saliens & 1.526 & 15,8 & 53 & 26,6 & 6231 & 15,8 & 45 & 26,2 & 2 & $<1,0$ & 1 & 1,9 & 7.759 & 14,3 & 99 & 23,3 \\
\hline Oligoplites saurus & 10 & $<1,0$ & 5 & 2.5 & 1.112 & 2.8 & 17 & 9,9 & 4 & $<1,0$ & 3 & 5,6 & 1.126 & 2.1 & 25 & 5,9 \\
\hline Ophioscion punctatissimus & 4 & $<1,0$ & 3 & 1,5 & 32 & $<1,0$ & 6 & 3.5 & 11 & $<1,0$ & 3 & 5,6 & 47 & $<1,0$ & 12 & 2.8 \\
\hline Opisthonema oglinum & & & & & 677 & 1,7 & 5 & 2.9 & & & inta & & 677 & 1,2 & 5 & 1,2 \\
\hline Orthopristis ruber & & & & & 4 & $<1,0$ & 2 & 1,2 & 3 & $<1,0$ & 3 & 5.6 & 7 & $<1,0$ & 5 & 1,2 \\
\hline Paralonchurus brasiliensis & & & & & 1 & $<1,0$ & 1 & $<1,0$ & 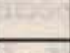 & Fr & whe & 272 & 1 & $<1,0$ & 1 & $\leq 1,0$ \\
\hline Pellona harroweri & - & & & & $a^{2}$ & & $\ln x$ & $C_{0}$ & 17 & $<1,0$ & 5 & 9,3 & 17 & $<1,0$ & 5 & 1.2 \\
\hline Percophis brasiliensis & & & & & 1 & $<1,0$ & 1 & $<1,0$ & & $18+5$ & & & 1 & $<1,0$ & 1 & $<1,0$ \\
\hline Phalloptychus januarius & & & & & 3 & $<1,0$ & 2 & 1,2 & & tin & $k$ & 2 & 3 & $<1,0$ & 2 & $<1,0$ \\
\hline Platanichthys platana & & & & & 7 & $<1,0$ & 3 & 1.7 & & & & & 7 & $<1,0$ & 3 & $<1,0$ \\
\hline Poecilia vivipara & 1 & $<1,0$ & 1 & $<1,0$ & 13 & $<1,0$ & 6 & 3,5 & & & & & 14 & $<1,0$ & 7 & 1,6 \\
\hline Polydactylus oligodon & & & 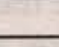 & & & & & & 1 & $<1,0$ & 1 & 1,9 & 1 & $<1,0$ & 1 & $\leq 1,0$ \\
\hline Polydactylus virginicus & 7 & $<1,0$ & 4 & 2,0 & 9 & $<1,0$ & 3 & 1,7 & 6 & $<1,0$ & 4 & 7.4 & 22 & $<1,0$ & 11 & 2,6 \\
\hline Pomadasys corvinaeformis & 21 & $<1,0$ & 6 & 3,0 & 29 & $<1,0$ & 10 & 5,8 & 41 & $<1,0$ & 11 & 20,4 & 91 & $<1,0$ & 27 & 6,4 \\
\hline Pomadasys croco & $=$ & & & & 37 & $<1,0$ & 1 & $<1,0$ & & & & 14 & 37 & $<1,0$ & 1 & $\leq 1,0$ \\
\hline Pomatomus saltator & 32 & $<1,0$ & 10 & 5,0 & 91 & $<1,0$ & 16 & 9,3 & 1 & $<1,0$ & 1 & 1,9 & 124 & $<1,0$ & 27 & 6,4 \\
\hline Porichthys porosissimus & & 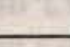 & & & 1 & $<1,0$ & 1 & $<1,0$ & $t 0$ & & $w$ & & 1 & $<1,0$ & 1 & $<1,0$ \\
\hline Prionotus punctatus & & 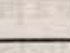 & & & & 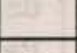 & 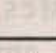 & $\therefore$ & 4 & $<1,0$ & 4 & 7.4 & 4 & $<1,0$ & 4 & $<1,0$ \\
\hline Rachycentron canadus & & & & & 1 & $<1,0$ & 1 & $<1,0$ & & 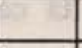 & - & ant & 1 & $<1,0$ & 1 & $<1,0$ \\
\hline Raneya fuminensis & & & & & ( & 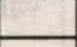 & & & 2 & $<1,0$ & 2 & 3.7 & 2 & $<1,0$ & 2 & $\leq 1,0$ \\
\hline Rivulus santensis & & & & & 1 & $<1,0$ & 1 & $<1,0$ & & & & & 1 & $<1,0$ & 1 & $\leq 1,0$ \\
\hline Sardinella brasiliensis & 4 & $<1,0$ & 3 & 1,5 & & & & & 1 & $<1,0$ & 1 & 1,9 & 5 & $<1,0$ & 4 & $<1,0$ \\
\hline Scorpaena plumieri & & & & & 1 & $<1,0$ & 1 & $<1,0$ & & & & & 1 & $<1,0$ & 1 & $\leq 1,0$ \\
\hline Selene setapinnis & & & & & & & & & 4 & $<1,0$ & 3 & 5,6 & 4 & $<1,0$ & 3 & $\leq 1,0$ \\
\hline Selene vomer & 1 & $<1,0$ & 1 & $\leq 1,0$ & 8 & $<1,0$ & 8 & 4,7 & 34 & $<1,0$ & 9 & 16,7 & 43 & $<1,0$ & 18 & 4.24 \\
\hline Sphoeroides greeleyi & 2 & $<1,0$ & 1 & $<1,0$ & 112 & $<1,0$ & 18 & 10,5 & 35 & $<1,0$ & 11 & 20,4 & 149 & $<1,0$ & 30 & 7,1 \\
\hline Sphoeroides testudineus & 3 & $<1,0$ & 3 & 1,5 & 81 & $<1,0$ & 9 & 5,2 & & + & & & 84 & $<1,0$ & 12 & 2.8 \\
\hline Sphyraena tome & & & & & 1 & $<1,0$ & 1 & $<1,0$ & 7 & $<1,0$ & 2 & 3.7 & 8 & $<1,0$ & 3 & $\leq 1,0$ \\
\hline Stellifer brasiliensis & & & & & 2 & $<1,0$ & 2 & 1,2 & & & & & 2 & $<1,0$ & 2 & $<1,0$ \\
\hline Stellifer rastrifer & 1 & $<1,0$ & 1 & $<1,0$ & 13 & $<1,0$ & 6 & 3,5 & & & & & 14 & $<1,0$ & 7 & 1,6 \\
\hline Stellifer stellifer & & & & & & & & & 1 & $<1,0$ & 1 & 1.9 & 1 & $<1,0$ & 1 & $\leq 1,0$ \\
\hline Strongylura marina & & & & & 1 & $<1,0$ & 1 & $<1,0$ & & & - & 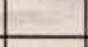 & 1 & $<1,0$ & 1 & $\leq 1,0$ \\
\hline Strongylura timucu & & & & & 3 & $<1,0$ & 3 & 1,7 & 21 & $<1,0$ & 8 & 14,8 & 24 & $<1,0$ & 11 & 2,6 \\
\hline Syacium papillosum & & & & & 11 & $<1,0$ & 5 & 2,9 & & & & & 11 & $<1,0$ & 5 & 1,2 \\
\hline Syngnathus dunckeri & 7 & $<1,0$ & 4 & 2,0 & & & & & & & & & 7 & $<1,0$ & 4 & $\leq 1,0$ \\
\hline Syngnathus folletti & 4 & $<1,0$ & 4 & 2,0 & 5 & $<1,0$ & 5 & 2,9 & 1 & $<1,0$ & 1 & 1,9 & 10 & $<1,0$ & 10 & 2.4 \\
\hline Synodus foetens & & & & & 1 & $<1,0$ & 1 & $<1,0$ & 1 & $<1,0$ & 1 & 1,9 & 1 & $<1,0$ & 1 & $<1,0$ \\
\hline Thyrsitops lepidopoides & & & & & 2 & $<1,0$ & 1 & $<1,0$ & & & & & 2 & $<1,0$ & 1 & $<1,0$ \\
\hline Trachinotus carolinus & 1.351 & 14.0 & 102 & 51,3 & 1.452 & 3.7 & 86 & 50,0 & 779 & 14,7 & 34 & 63,0 & 3.582 & 6.6 & 222 & 52,2 \\
\hline Trachinotus falcatus & 15 & $<1,0$ & 12 & 6,0 & 515 & 1,3 & 54 & 31,4 & 277 & 5,2 & 24 & 44,4 & 807 & 1,5 & 90 & 21,2 \\
\hline Trachinotus goodel & 1.171 & 12.1 & 136 & 68,3 & 569 & 1,4 & 64 & 37,2 & 74 & 1.4 & 19 & 35,2 & 1.814 & 3,3 & 219 & 51,5 \\
\hline Trachinotus marginatus & 9 & $<1,0$ & 7 & 3,5 & & & & & & & & & 9 & $<1,0$ & 7 & 1,6 \\
\hline Umbrina coroides & 8 & $<1,0$ & 4 & 2,0 & 39 & $<1,0$ & 17 & 9,9 & 1.803 & 33.9 & 25 & 46,3 & 1.850 & 3,4 & 46 & 10,8 \\
\hline Xenomelaniris brasiliensis & 178 & 1,8 & 39 & 19,6 & 2449 & 6.2 & 90 & 52,3 & 810 & 15,2 & 40 & 74,1 & 3.437 & 6,3 & 169 & 39,8 \\
\hline TOTAL & 9.650 & 100 & & & 39.418 & 100 & & & 5.316 & 100 & & & 54.384 & 100 & & \\
\hline+20 & 56 esp. & 199 & & 7ar & 67 esp & 172 & m. & 6 ar. & 52 esp & 54 & & 162 at & 99 esp & & & 275 ar \\
\hline
\end{tabular}


Um total de 99 espécies pertencentes a 73 gêneros e a 40 famílias ocorreu nas áreas de estudo do presente trabalho, sendo 56 espécies (21 familias), com uma média de captura pouco superior a 16 indivíduos por arrasto no litoral sul, 67 espécies ( 31 famílias) com uma média de captura superior a 76 indivíduos por arrasto no litoral central, e 52 espécies ( 25 famílias), com uma média de captura pouco inferior a 33 indivíduos por arrasto no litoral norte (Tab. 1).

Carangidae, Sciaenidae, Mugilidae, Clupeidae, Atherinidae, Engraulididae e Ariidae foram as famílias mais abundantes no litoral sul, sendo as duas primeiras as que apresentaram maior número de espécies (Fig. 3). Oligoplites saliens, Trachinotus carolinus, Trachinotus goodei, Menticirrhus littoralis e Harengula clupeola formaram o grupo das espécies mais abundantes, correspondendo a mais de $74 \%$ da captura total em número (Tab. 1).

Engraulididae, Carangidae, Mugilidae, Sciaenidae, Atherinidae, Clupeidae e Ariidae foram as famílias mais abundantes no litoral central, sendo que Carangidae e Sciaenidae foram as que apresentaram maior número de espécies (Fig. 3). Anchoviella lepidentostole, Oligoplites saliens, Mugil curema, Menticirrhus littoralis e Mugil gaimardianus foram as espécies mais abundantes, correspondendo a mais de $66 \%$ da captura total em número (Tab. 1).
Sciaenidae, Carangidae, Atherinidae, Engraulididae, Clupeidae, Sparidae e Dactyloscopidae foram as famílias mais abundantes no litoral norte, sendo as duas primeiras as que apresentaram maior número de espécies (Fig. 3). Umbrina coroides, Xenomelaniris brasiliensis, Trachinotus carolinus, Anchoa tricolor e Trachinotus falcatus foram as espécies mais abundantes, correspondendo a mais de $78 \%$ da captura total em número (Tab. 1).

As espécies estiveram representadas por indivíduos cuja amplitude de comprimentos variou entre 11 e $240 \mathrm{~mm}$ de comprimento total. A menor amplitude de comprimento foi registrada no litoral sul e a maior no litoral norte.

O padrão sazonal da captura por unidade de esforço foi o mesmo para as três áreas, com maiores valores no verão-outono e menores no inverno-primavera, sendo as capturas bem superiores no litoral central, em comparação às demais áreas. Embora o número de amostras efetuadas no litoral norte tenha sido o menor, as capturas nesta área foram superiores às do litoral sul, área onde o esforço de pesca foi o maior (Fig. 4).

Quanto aos índices de estrutura de comunidades, observou-se, para a área sul, maiores valores de S, D e H' durante o verāo e de J' durante o outono, e menores valores de $\mathbf{D}, \mathbf{H}$ ' e J' durante a primavera e de $\mathbf{S}$ durante o inverno. Para a área central, todos os índices apresentaram maiores valores durante o outono e menores na primavera. Para a

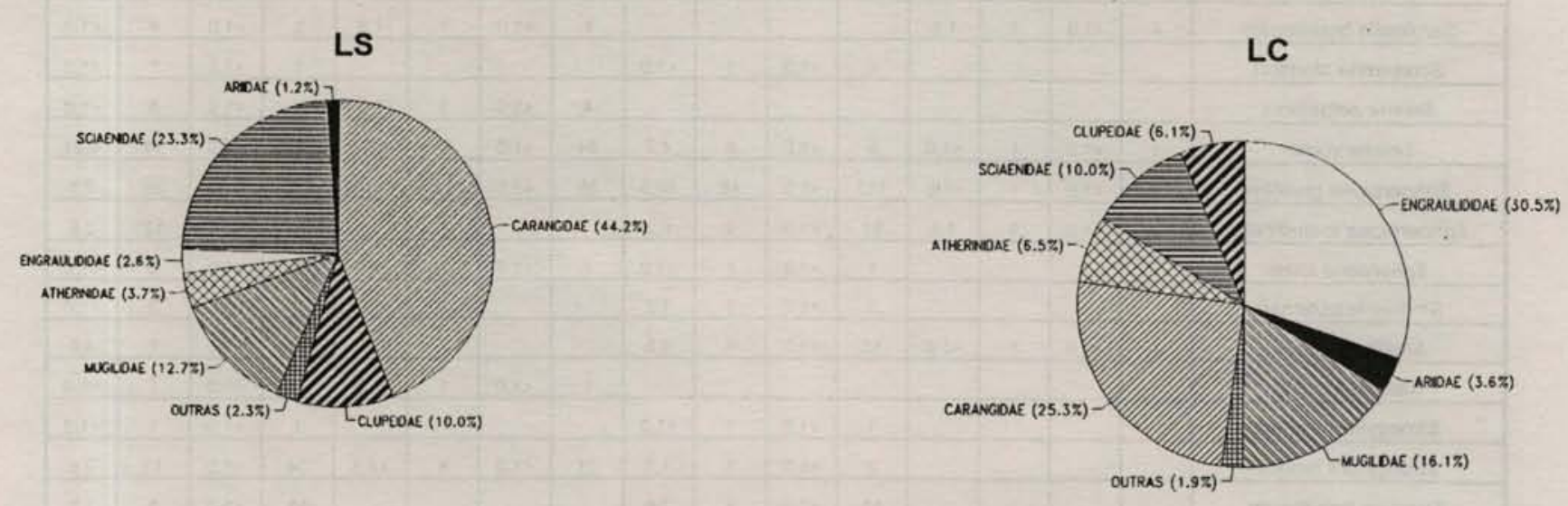

LN

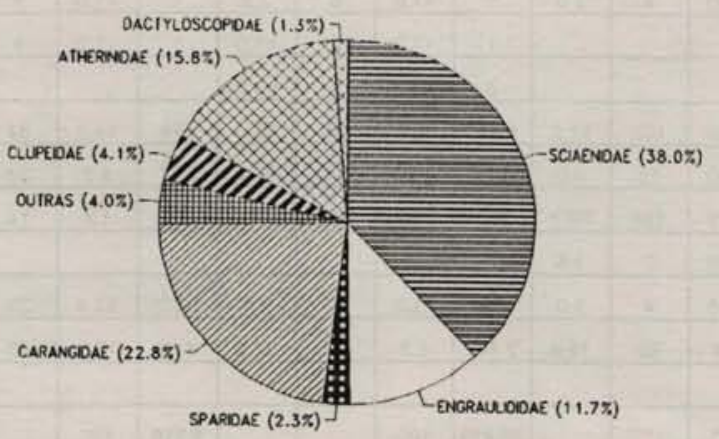

Fig. 3. Percentual em número das familias mais abundantes no litoral sul (LS), central (LC) e norte (LN). 


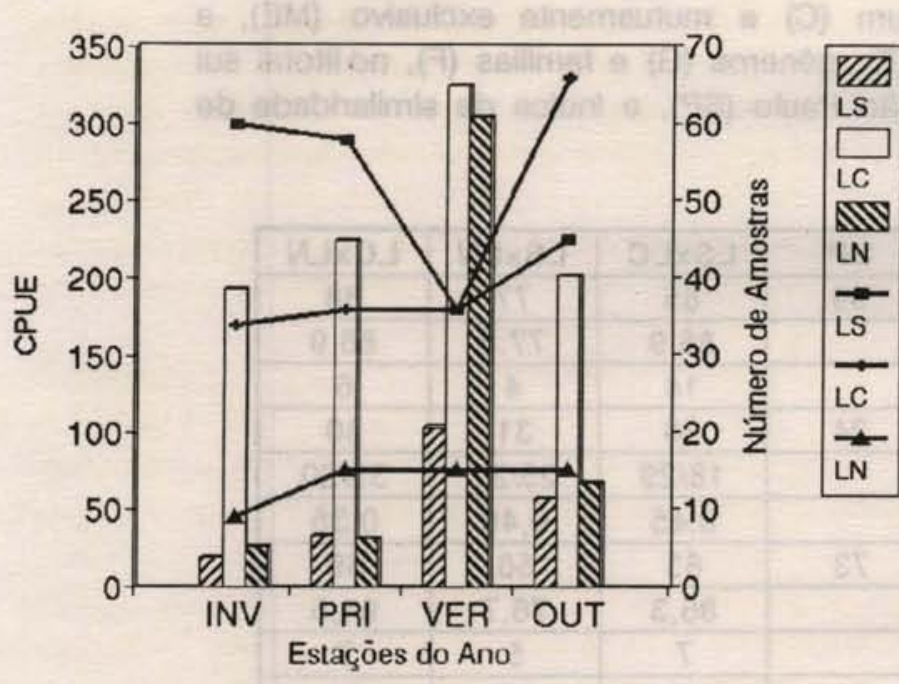

Fig. 4. Variação sazonal da abundância relativa, expressa em CPUE (barra), e do número de amostras efetuadas (linha cheia), no litoral sul (LS), central (LC) e norte (LN).

área norte, observou-se maiores valores de S, D e $\mathbf{H}^{\prime}$ durante a primavera e de $\mathbf{J}$ ' durante $o$ inverno, e menores valores de $\mathbf{D}, \mathbf{H}$ ' e J' durante o verão e de $\mathbf{S}$ durante o inverno (Fig. 5).

Maior número de espécies, gêneros, famílias e de indivíduos por amostra foi registrado para o litoral central; para um número ligeiramente inferior de amostras realizadas, em comparação com o litoral sul (172:199), sua abundância relativa média foi quase que quadruplicada. No litoral norte, apesar do baixo número de amostras realizadas em comparação com o litoral sul, amostramos um maior número de gêneros e famílias e a abundância relativa foi o dobro desta (Tabs 1 e 2).

Considerando-se a área como um todo, 24 espécies, 21 gêneros e 14 famílias, correspondendo respectivamente, a $24,2 \%, 28,8 \%$ e $35,0 \%$ do total dos táxons, foram comuns; deste total, 14 espécies, 13 gêneros e 7 famílias corresponderam a menos de $1,0 \%$, cada um, da abundância total (Tabs 1 e 2).

O número de táxons exclusivos de cada uma das áreas, bem como entre as áreas, aumentou do nível de família ao nível de espécie, sendo que o litoral central (LC) e o litoral sul (LS) apresentaram, respectivamente, o maior e o menor número de táxons exclusivos, em qualquer nível; a percentagem de táxons exclusivos de uma área variou entre 5,0 e $25,0 \%$ (Tab. 2).

Entre as áreas, o número de táxons exclusivos foi maior entre o litoral sul e o litoral central e menor entre o litoral sul e o litoral norte; a percentagem de táxons exclusivos variou entre 5,2 e $16,5 \%$ (Tab. 2 ).

No litoral sul, nenhum dos táxons exclusivos foi abundante; no litoral central foram abundantes somente a nível de espécie, Anchoa filifera, Opisthonema oglinum e Eucinostomus gula, e no litoral norte, foram abundantes Diplodus argenteus e Dactyloscopus tridigitatus.
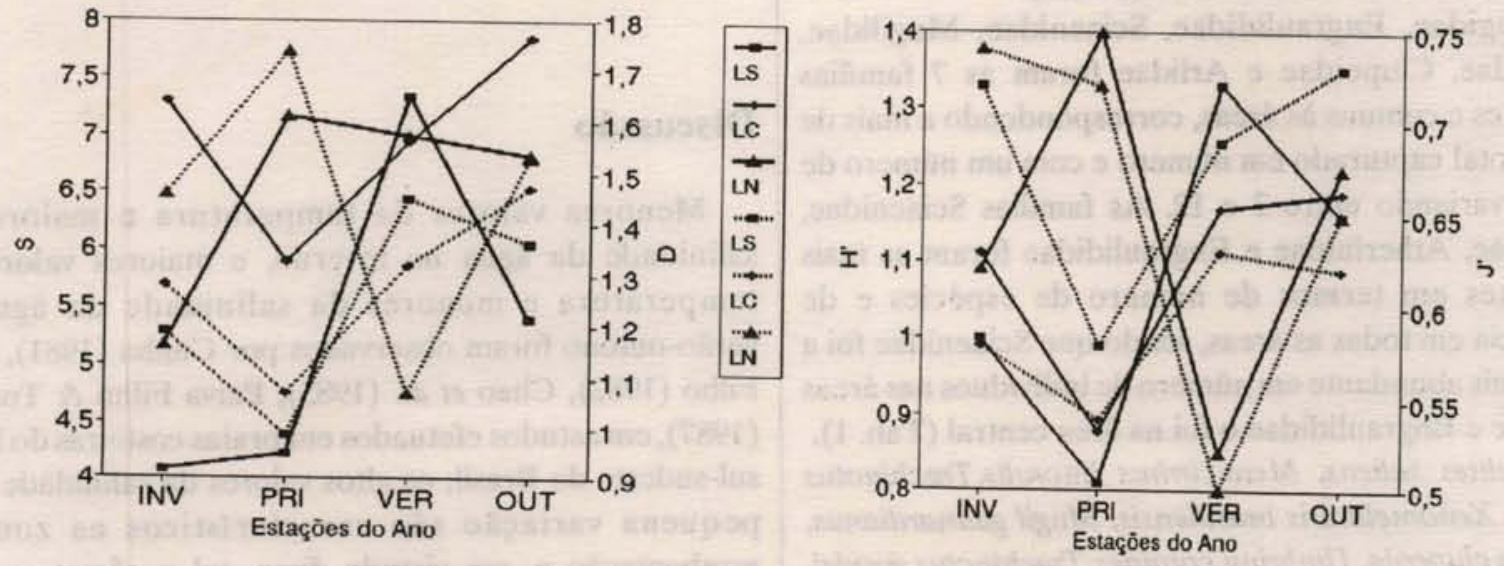

Fig. 5. Variação sazonal do número médio de espécies $\mathbf{S}$ (linha cheia), e dos índices de riqueza de espécies D (linha pontilhada), de diversidade de Shannon H' (linha cheia), e de eqüitabilidade J' (linha pontilhada), no litoral sul (LS), central (LC) e norte (LN). 
Tabela 2. Número total (T), exclusivo (E), comum (C) e mutuamente exclusivo (ME), e percentagem do número total $(\%)$ de espécies $(E)$, gêneros $(G)$ e familias $(F)$, no litoral sul (LS), central (LC), norte (LN) e no Estado de São Paulo (SP), e indice de similaridade de Jaccard (IJ) entre as áreas

\begin{tabular}{|c|c|c|c|c|c|c|c|}
\hline & LS & LC & LN & SP & LSxLC & LSxLN & LCxLN \\
\hline ET & 56 & 67 & 52 & 99 & 85 & 77 & 88 \\
\hline$\% E$ & & & & & 85,9 & 77,8 & 88,9 \\
\hline EE & 11 & 22 & 14 & & 14 & 4 & 6 \\
\hline EC & & & & 24 & 38 & 31 & 30 \\
\hline EME & & & & & $18 / 29$ & $25 / 21$ & $35 / 20$ \\
\hline IJ & & & & & 0,45 & 0,40 & 0,35 \\
\hline GT & 39 & 54 & 43 & 73 & 63 & 56 & 69 \\
\hline$\% G$ & & & & & 86,3 & 76,7 & 94,5 \\
\hline GE & 4 & 17 & 10 & & 7 & 5 & 7 \\
\hline GC & & & & 21 & 30 & 26 & 28 \\
\hline GME & & & & & $9 / 24$ & $13 / 17$ & $26 / 15$ \\
\hline IJ & & & & & 0,48 & 0,46 & 0,41 \\
\hline FT & 21 & 31 & 25 & 40 & 35 & 30 & 38 \\
\hline$\% F$ & & & & & 87,5 & 75,0 & 95,0 \\
\hline FE & 2 & 10 & 5 & & 3 & 2 & 4 \\
\hline FC & & & & 14 & 17 & 16 & 18 \\
\hline FME & & & & & $4 / 14$ & $5 / 9$ & $13 / 7$ \\
\hline IJ & & & & & 0,49 & 0,53 & 0,47 \\
\hline
\end{tabular}

A similaridade faunal, ainda que semelhante, em todas as áreas aumentou do nível de espécie ao nível de família. Entre as áreas e a cada nível taxonômico, a similaridade faunística foi menor entre o litoral central e o litoral norte e maior entre o litoral sul e o litoral central, com exceção ao nível de família, onde a maior similaridade foi verificada entre o litoral sul e o litoral norte (Tab. 2).

Carangidae, Engraulididae, Sciaenidae, Mugilidae, Atherinidae, Clupeidae e Ariidae foram as 7 famílias abundantes e comuns às áreas, correspondendo a mais de $97 \%$ do total capturado em número e com um número de espécies variando entre 2 e 12. As famílias Sciaenidae, Carangidae, Atherinidae e Engraulididae foram as mais abundantes em termos de número de espécies e de abundância em todas as áreas, sendo que Sciaenidae foi a família mais abundante em número de indivíduos nas áreas sul e norte e Engraulididae o foi na área central (Tab. 1).

Oligoplites saliens, Menticirrhus littoralis, Trachinotus carolinus, Xenomelaniris brasiliensis, Mugil gaimardianus, Harengula clupeola, Umbrina coroides, Trachinotus goodei, Oligoplites saurus e Trachinotus falcatus foram as 10 espécies abundantes e comuns às áreas, correspondendo a mais de $58 \%$ do total capturado em número (Tab. 1).

Entre as áreas foram comuns trinta gêneros e dezessete famílias, compreendendo entre 1 a 4 espécies por gênero e 1 e 11 espécies por família, entre o litoral sul e o litoral central, vinte e seis gêneros e dezesseis famílias, compreendendo entre 1 a 4 espécies por gênero e 1 e 11 espécies por família, entre o litoral sul e o litoral norte, e vinte e oito gêneros e dezoito famílias, compreendendo entre 1 a 3 espécies por gênero e 1 e 9 espécies por família, entre o litoral central e o litoral norte (Tabs $1 \mathrm{e} 2$ ).

\section{Discussão}

Menores valores da temperatura e maiores da salinidade da água no inverno, e maiores valores da temperatura e menores da salinidade da água no verāo-outono foram observados por Cunha (1981), Paiva Filho (1982), Chao et al. (1985), Paiva Filho \& Toscano (1987), em estudos efetuados em praias costeiras do litoral sul-sudeste do Brasil; os altos valores da salinidade e sua pequena variação são característicos na zona de arrebentação e, em virtude disso, tal parâmetro não é considerado um fator importante na organizaçāo da estrutura comunitária nesse tipo de ambiente.

Maiores valores da temperatura da água, superiores à temperatura do ar, principalmente durante o verão, foram relatados nos estudos de Cunha (1981) e Chao et al. (1985). Menores valores da temperatura da água em relação à 
temperatura atmosférica verificados na primavera e início de verāo no litoral norte, relatados também por Cunningham (1983), estâo relacionados com a presença marcante da Água Central do Atlântico Sul (ACAS), que nessa época do ano ocasiona o surgimento de uma termoclina ao largo da região de Ubatuba (Castro Filho et al., 1987).

Maiores valores da abundância no verāo-outono e menores no inverno foram descritos em vários estudos, como os de Anderson et al. (1977), Matsuura \& Nakatani (1979), Cunha (1981), Modde \& Ross (1981), Paiva Filho (1982) e Saul (1994).

No litoral sul, Zani-Teixeira (1983), estudando a ictiofauna demersal, encontrou Carangidae e Sciaenidae como familias abundantes e de maior número de espécies, sendo $O$. saliens uma das espécies mais abundantes. Saul (1994), estudando a ictiofauna da zona de arrebentaçāo da Ilha de Bom Abrigo, situada ao largo da regiâo de Cananéia, encontrou Carangidae e Clupeidae como as famílias mais expressivas em número de espécies, tendo sido Harengula clupeola a espécie mais abundante. Acreditamos que as discrepâncias dos nossos resultados em relação aos de Saul (1994), principalmente pela inexpressividade de captura de $O$. saliens e $T$. carolinus, espécies abundantes no presente estudo, estão relacionadas com o baixo rendimento obtido pela autora, já que o número total de espécies (18), famílias (12) e indivíduos capturados (400) foi muito baixo.

Praticamente a mesma ordem de abundância nas famílias foi verificada por Paiva Filho (1982) e por Paiva Filho \& Toscano (1987) em Santos e São Vicente, os quais relatam como as espécies mais abundantes nos arrastos de praia da região os mugilídeos, Trachinotus carolinus, $T$. falcatus eXenomelaniris brasiliensis. Estudando a fauna de peixes demersais, Ribeiro Neto (1989) capturou 92 espécies com arrastos de fundo na Baía de Santos no mesmo período, tendo sido Sciaenidae a família mais abundante em número de espécies e de indivíduos, seguida de Clupeidae e de Ariidae.

Cunningham (1983) e Rocha (1990), estudando a comunidade de peixes demersais da regiāo de Ubatuba, observaram que a família mais importante (número de espécies, abundância e fidelidade) foi Sciaenidae. $U$. coroides foi a larva mais abundante e freqüente no estudo de Matsuura \& Nakatani (1979) e Trachinotus carolinus foi um dos carangídeos mais abundantes e freqüentes no estudo de Scorvo Filho et al. (1987).

A predominância de exemplares pequenos, maiores abundâncias nos meses mais quentes do ano e poucas espécies dominantes são características comuns da ictiofauna da zona de arrebentação, conforme revelam os dados de diversos autores (Anderson Jr. et al., 1977; Matsuura \& Nakatani, 1979; Saloman \& Naughton, 1979;
Cunha, 1981; Modde \& Ross, 1981; Paiva Filho \& Toscano, 1987).

Para a ictiofauna da Ilha do Bom Abrigo, Saul (1994) observou maiores valores do número de espécies e da riqueza na primavera, e dos índices de diversidade e equitatividade no outono-inverno. Para Paiva Filho \& Toscano (1987), a diversidade no litoral central foi maior no outono e menor na primavera em correlação com a salinidade, e Ribeiro Neto (1989) encontrou o mesmo padrão para os índices de diversidade da fauna de peixes demersais da Baía de Santos.

Para o litoral norte, Rocha (1990) e Rossi-Wongtschowski \& Paes (1993) observaram, em relaçāo à comunidade de peixes demersais, maiores valores da riqueza de espécies durante o inverno. Os baixos valores de riqueza de espécies, diversidade e eqüitabilidade durante o verão observados no presente estudo estão correlacionados, possivelmente, com a grande abundância de Umbrina coroides nesta época, já que a dominância de poucas espécies influencia negativamente os valores dos índices de diversidade $\mathrm{e}$ eqüitatividade.

Saloman \& Naughton (1979) e Cunha (1981) observaram maior diversidade no verâo e menor na primavera, sendo o número de espécies maior na primavera-verâo-outono e menor no inverno.

Ribeiro Neto (1989) comparou os litorais sul, central e norte e concluiu que o sul era mais abundante mas apresentava o menor número de espécies, e o norte era o menos abundante mas apresentava o maior número de espécies.

Podemos tecer algumas hipóteses para a maior abundância numérica e taxonômica (em todos os níveis) no litoral central. Num primeiro momento, em se comparando o litoral central com o litoral sul, e considerando que ambos apresentam feiçōes fisiográficas semelhantes (extensa faixa de praia arenosa contínua, influência de grande aporte de água doce, presença de estuários e manguezais), deveríamos supor que os maiores valores da abundância e de táxons ocorreriam no litoral sul, já que este é, comprovadamente, nada ou muito pouco alterado por atividades antrópicas.

Contudo, ocorreu o inverso e tal fato deve estar relacionado com o ambiente altamente eutrofizado que é a Baía de Santos (Gianesella-Galvão, 1978; Tommasi, 1979, 1987; CETESB, 1985). McFarland (1963) salienta que a maioria das espécies de peixes da zona de arrebentação é planctófaga e, assim sendo, em regiōes climáticas afins, áreas mais produtivas tendem a apresentar maior número de espécies; há uma nítida relação entre a disponibilidade de alimento e a diversidade de espécies, sendo que um aumento na quantidade de alimento favorece uma maior especialização alimentar por parte das espécies e, conseqüentemente, uma maior 
repartição dos recursos, com aumento final do estoque pesqueiro (Cushing, 1975).

Outros fatores também importantes podem estar relacionados com o grau de exposição das praias, já que a Baía de Santos atua como uma barreira, amenizando o impacto das correntes marinhas e com uma maior heterogeneidade ambiental, pela presença de costões rochosos limitando as praias, o que nāo ocorre com o litoral sul; locais protegidos, de baixa energia e com fluxo de água suave apresentam maior número de espécies que locais agitados com ondas fortes.

Ainda que o número de amostras efetuadas no litoral norte tenha sido inferior a $1 / 3$ do número de amostras efetuadas no litoral sul, o fator heterogeneidade ambiental deve também ter sido o responsável pelos maiores valores no número de indivíduos, gêneros e famílias verificados no litoral norte em comparaçāo com o litoral sul. Devemos considerar, contudo, que estes maiores valores no litoral norte podem estar relacionados com o fato de que o litoral ao largo de Ubatuba é, comprovadamente, local de desova de várias espécies de peixes no inverno e verão, destacando-se principalmente Carangidae, Clupeidae, Engraulididae e Mugilidae (Katsuragawa et al., 1993).

A predominância da família Carangidae, tanto em número de indivíduos, quanto em número de espécies, está relacionada com o fato de que os juvenis desta família acham-se associados à zona de arrebentação de praias, que é o ambiente natural e mais propício ao desenvolvimento dos primeiros estágios de vida (Cunha, 1987, 1990).

Finalmente, devemos salientar a importância do conhecimento da comunidade ictiofaunística da zona de arrebentação; sua análise contribui enormemente não só para o conhecimento das inúmeras espécies que ocorrem neste ambiente, mas também para a compreensão dos mecanismos de repartição que permitem que várias espécies coexistam nos ambientes adjacentes e, preliminarmente, melhora a caracterização da composição da ictiofauna de uma região, como bem demonstrado nos trabalhos de Chao et al. (1982) e Paiva Filho et al. (1987), já que várias espécies de peixes ocorrem exclusivamente na zona de arrebentação. Nossos resultados acrescentam 30 espécies à comunidade de peixes do litoral sul (Zani-Teixeira, 1983), 27 espécies à comunidade de peixes do litoral central (Ribeiro Neto, 1989), e entre 22 e 27 espécies à comunidade de peixes do litoral norte (Cunningham, 1983; Rocha, 1990), sendo que nenhuma das espécies consideradas como dominantes nos arrastos de fundo efetuados nos trabalhos acima citados, o foi para os arrastos de praia do presente estudo.

\section{Agradecimentos}

Aos amigos Francisco Borba Ribeiro Neto, pelo auxílio nos trabalhos de campo e de laboratório das amostras de
Santos; João Marcos Miragaia Schmiegelow, pelo auxílio nos trabalhos de campo das amostras de Santos e de Ubatuba; Roberto Ribas Gallucci, pelo auxílio nos trabalhos de campo e de laboratório das amostras de Ubatuba e Ilha Comprida e Ana Paula Toscano, pelo auxílio nos trabalhos de campo e de laboratório das amostras de Ubatuba.

Aos funcionários Sérgio C. Veiga, Clóvis, Joāo Xavier, Umberto Dias e Pércio Pereira, da Base de Cananéia do Instituto Oceanográfico, Oscar Barbosa e Edgar Borges, da Base de Santos do Instituto Oceanográfico, pela colaboração nas coletas das amostras da Ilha Comprida e de Santos, respectivamente.

\section{Referências bibliográficas}

ANDERSON JR, W. D.; DIAS, J. K.; DIAS, R. K.; CUPKA, D. M. \& CHAMBERLAIN, N. A. 1977. The macrofauna of the surf zone off Folly Beach, South Carolina. Spec. scient. Rept. Fisheries Bull natn. mar. Fish. Serv., U.S., (74):1-23.

BROWN, A. C. \& McLACHLAN, A. 1990. Ecology of sandy shores. New York, Elsevier. 328p.

CASTRO FILHO, B. M. de; MIRANDA, L. B. de \& MIYAO, S. Y. 1987. Condiçōes oceanográficas na plataforma continental ao largo de Ubatuba: variaçōes sazonais e em média escala. Bolm Inst. oceanogr., S Paulo, 35(2):135-151.

CETESB. 1985. Baixada Santista, carta do meio ambiente e de sua dinâmica. Metodologia do Prof. André Journaux. Memorial descritivo. São Paulo, CETESB. 33p.

CHAO, L. N.; PEREIRA, L. E; VIEIRA, J. P. BEMVENUTI, M. A. \& CUNHA, L. P. R. 1982. Relação preliminar dos peixes estuarinos e marinhos da Lagoa dos Patos e regiâo costeira adjacente, Rio Grande do Sul, Brasil. Atlântica, Rio Grande, 5(1):67-75.

\&

1985. Estuarine fish community of the Patos Lagoon, Brazil - a baseline study. In: Yáñez-Arancíbia, A. ed. Fish community ecology in estuaries and coastal lagoons-towards an ecosystem integration. UNAM Press (DR), México. p.429-450. 
CUNHA, L.P. R. 1981. Variação sazonal da distribuição, abundância e diversidade dos peixes na zona de arrebentação da praia do Cassino, R.S. - Brasil. Dissertação de mestrado. Universidade Federal do Rio de Janeiro. 47p.

1987. Importância da zona de arrebentação de praias para o desenvolvimento dos juvenis de Trachinotus (Pisces, Carangidae): Aspectos da bioecologia e distribuição geográfica do gênero, com ênfase às espécies que ocorrem no litoral Sul/Sudeste do Brasil e no Atlântico Ocidental. Tese de doutorado. Universidade de São Paulo, Instituto de Biociências. 250p.

1990. Sobre a distribuição geográfica de Trachinotus goodei Jordan \& Evermann, 1896 (Teleostei-Carangidae). In: XVII CONGRESSO BRASILEIRO DE ZOOLOGIA. Londrina, 1990. Resumos. Londrina, Sociedade Brasileira de Zoologia, p. 285.

CUNNINGHAM, P. T. M. 1983. Estudo comparativo da ictiofauna da costa oeste e Enseada das Palmas da Itha Anchieta, Enseada do Flamengo e Enseada da Fortaleza (Lat. $23^{\circ} 29^{\prime} \mathrm{S}-23^{\circ} 33^{\prime} \mathrm{S}$, Long. $45^{\circ} 3^{\prime} \mathrm{W}-45^{\circ} 9^{\prime} \mathrm{W}$ ), Ubatuba, Estado de São Paulo Brasil. Tese de doutorado. Universidade de São Paulo, Instituto Oceanográfico. 133p.

CUSHING, D. H. 1975. Marine ecology and fisheries. Cambridge, Cambridge University Press. 278p.

FIGUEIREDO, J. L. 1977. Manual de peixes marinhos do sudeste do Brasil. I. Introdução. Caçōes, raias e quimeras. São Paulo, Museu de Zoologia da Universidade de São Paulo. 104p.

\& MENEZES, N. A. 1978. Manual de peixes marinhos do sudeste do Brasil. II. Teleostei (1). São Paulo, Museu de Zoologia da Universidade de São Paulo. 110p.

$$
\text { \& }
$$

1980. Manual de

peixes marinhos do sudeste do Brasil. III. Teleostei (2). São Paulo, Museu de Zoologia da Universidade de Sẫo Paulo. 90p.

FISCHER, W. ed. 1978. FAO species identification sheets for fishery purposes. Western Central Atlantic (fishing area 31). Roma, FAO. v. 1-7.
GIANESELlA-GALVÃo, S. M. F. 1978. Produção primária da Baía de Santos, Estado de São Paulo. Aspectos sobre a eficiência fotossintética num ambiente marinho poluído. Dissertação de mestrado. Universidade de São Paulo, Instituto Oceanográfico. 53p.

GIANNINI, R. 1994. Estrutura das comunidades de peixes da zona de arrebentação de praias arenosas do litoral do Estado de São Paulo, Brasil. Tese de doutorado. Universidade de São Paulo, Instituto Oceanográfico. 139p.

KATSURAGAWA, M.; MATSUURA, Y.; SUZUKI, K.; DIAS, J. F. \& SPACH, H. L. 1993. O ictioplâncton ao largo de Ubatuba, SP: composição, distribuição e ocorrência sazonal (1985-1988). In: Pires-Vanin, A. M. S., ed. Estrutura e função do ecossistema de plataforma continental do Atlântico Sul Brasileiro. Publção esp. Inst. oceanogr., S Paulo, (10):85-121.

McLACHLAN, A. \& ERASMUS, T. eds 1983. Sandy beaches as ecosystems. The Hague, Dr. W. Junk. 757p.

McFARLAND, W. N. 1963. Seasonal change in the number and biomass of fishes from the surf at Mustang Island, Texas. Publs Inst. mar. Sci Univ. Tex., 9:91-112.

MATSUURA, Y. \& NAKATANI, K. 1979. Ocorrência de larvas e jovens de peixes na Ilha Anchieta (SP), com algumas anotaçōes sobre a morfologia da castanha Umbrina coroides Cuvier, 1830. Bolm Inst. oceanogr., S Paulo, 28(1):165-183.

MENEZES, N. A. \& FIGUEIREDO, J. L. 1980. Manual de peixes marinhos do sudeste do Brasil. IV. Teleostei (3). São Paulo, Museu de Zoologia da Universidade de São Paulo. 96p.

$$
\text { \& }
$$
1985. Manual de peixes marinhos do sudeste do Brasil. V. Teleostei (4). São Paulo, Museu de Zoologia da Universidade de São Paulo. 105p.

MODDE, T. \& ROSS, S. T. 1981. Seasonality of fishes occupying a surf zone habitat in the northern Gulf of Mexico. Fishery Bull. natn. mar. Fish. Serv., U.S., 78(4):911-922. 
MONTEIRO NETO, C. 1990. Comparative community structure of surf- zone fishes in the Chesapeake Bight and Southern Brazil. Ph.D. Thesis. Virginia Institute of Marine Science, Faculty of the School of Marine Science. 150p.

NORMAN, J. R. 1934. A systematic monograph of the flatfishes (Heterosomata). Vol. 1. Psettodidae, Bothidae, Pleuronectidae. London, British Museum. v.1, p.59-261. (Reprint by Johnson Reprint Co.).

PAIVA FILHO, A. M. 1982. Estudo sobre a ictiofauna do Canal dos Barreiros, Estuário de São Vicente, SP. Tese de livre-docência. Universidade de São Paulo, Instituto Oceanográfico. 189p.

\& TOSCANO, A. P. 1987. Estudo comparativo e variação da ictiofauna na zona entre-marés do Mar Casado - Guarujá-Mar Pequeno-São Vicente, SP. Bolm Inst. oceanogr. S Paulo, 35(2):153-165.

\& GIANNINI, R.; RIBEIRO NETO, F. B. \& SCHMIEGELOW, J. M. M. 1987. Ictiofauna do complexo baía-estuário de Santos e Sāo Vicente, SP, Brasil. Relat. int. Inst. oceanogr. Univ. S Paulo, (17):1-10.

RIBEIRO NETO, F. B. 1989. Estudo da comunidade de peixes da Baía de Santos, SP. Dissertação de mestrado. Universidade de São Paulo, Instituto Oceanográfico. 196p.

ROCHA, G. R. A. 1990. Distribuiçāo, abundância e diversidade da ictiofauna na regiāo de Ubatuba - SP $\left(23^{\circ} 20^{\prime} \mathrm{S}-24^{\circ} 00^{\prime} \mathrm{S} ; 44^{\circ} 30^{\prime} \mathrm{W}-45^{\circ} 30^{\prime} \mathrm{W}\right)$, Brasil. Dissertação de mestrado. Universidade de São Paulo, Instituto Oceanográfico. 2v.

ROSSI-WONGTSCHOWSKI, C. L. D. B. \& PAES, E. T. 1993. Padrões espaciais da comunidade de peixes demersais do litoral norte do Estado de São Paulo-Ubatuba, Brasil. In: Pires-Vanin, A. M. S., ed. Estrutura e função do ecossistema de plataforma continental do Atlântico Sul Brasileiro. Publção esp. Inst. oceanogr., S Paulo, (10):169-188.
SALOMAN, C. H. \& NAUGHTON, S. P. 1979. Fishes of the littoral zone, Pinellas County, Florida. Fla. Scient., 42(2):85-93.

SAUL, A. de C. 1994. Comunidade ictiofaunística da Ilha do Bom Abrigo, Cananéia, São Paulo, Brasil. Dissertação de mestrado. Universidade de São Paulo, Instituto Oceanográfico. 118p.

SCORVO FILHO, J. D.; HORIKANA, M. T.; BARROS, H. P. de \& BASTOS, A. A. 1987. Identificação e ocorrência de alevinos de Trachinotus

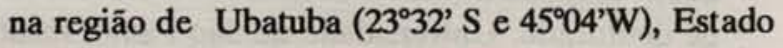
de São Paulo, Brasil. Bolm Inst. Pesca, S Paulo, 14:35-43.

TOMMASI, L. R. 1979. Consideraçōes ecologicas sobre o sistema estuarino de Santos (SP). Tese de livre-docência. Universidade de São Paulo, Instituto Oceanográfico. 2v.

1987. Poluição marinha no Brasil: síntese do conhecimento. Publção esp. Inst. oceanogr., Univ. S Paulo, (5):1-30.

ZANI-TEIXEIRA, M. de L. 1983. Contribuição ao conhecimento da ictiofauna da Baía de Trapandé, complexo estuarino-lagunar de Cananéia. Dissertação de mestrado. Universidade de São Paulo, Instituto Oceanográfico. 83p.

(Manuscrito recebido 17 outubro 1995, aceito 9 novembro 1995) 